\title{
Ray Tracing Simulation in Nonspherically Symmetric Atmosphere for GPS Radio Occultation
}

\author{
Wen-Hao Yeh ${ }^{1,2}$, Cheng-Yung Huang ${ }^{1, *}$, Tsen-Chieh Chiu ${ }^{2}$, Ming-Quey Chen ${ }^{3}$, Jann-Yenq Liu ${ }^{3,4,5}$, and \\ Yuei-An Liou ${ }^{4}$ \\ ${ }^{1}$ GPS Science and Application Research Center, National Central University, Taoyuan, Taiwan \\ ${ }^{2}$ Department of Electrical Engineering, National Central University, Taoyuan, Taiwan \\ ${ }^{3}$ Graduate Institute of Space Science, National Central University, Taoyuan, Taiwan \\ ${ }^{4}$ Center for Space and Remote Sensing Research, National Central University, Taoyuan, Taiwan \\ ${ }^{5}$ National Space Organization, Hsinchu, Taiwan
}

Received 26 September 2013, revised 7 July 2014, accepted 7 July 2014

\begin{abstract}
A three-dimensional ray tracing model with aiming algorithms for global positioning system (GPS) signal is proposed to make simulations conform to the realistic radio occultation (RO) signal propagation. The two aiming algorithms used in this study ensure the initial and end point ray trajectories are located in the prescribed region. In past studies, the ray tracing techniques applied to the RO signal simulation usually assumed a spherically symmetrical atmosphere for simplicity. The exact GPS and low earth orbit (LEO) satellite locations are not considered in the simulation. These two assumptions make the simulation unrealistic for GPS signal propagation in the RO technique. In the proposed model, the shape of the earth is assumed as an ellipse. The information from European Centre for Medium-Range Weather Forecasts (ECMWF) analysis is used to setup the atmosphere in the simulation. Two aiming algorithms are developed to determine the initial signal propagating direction to make the simulated signal start from the prescribed GPS satellite position and end in the close vicinity of the LEO satellite position. An ideal spherical symmetric atmospheric structure is used to verify the ray tracing model. The fractional difference between real and simulated refractivity results is less than $0.1 \%$. Otherwise, the GPS and LEO satellite position in the Formosat-3/COSMIC observation and the ECMWF analysis, considering the earth's flattening, is also used to verify the aiming algorithms. All of the simulated signals end in close vicinity to the LEO satellite position in the simulation results.
\end{abstract}

Key words: Radio occultation, Ray tracing, ECMWF

Citation: Yeh, W. H., C. Y. Huang, T. C. Chiu, M. Q. Chen, J. Y. Liu, and Y. A. Liou, 2014: Ray tracing simulation in nonspherically symmetric atmosphere for GPS radio occultation. Terr. Atmos. Ocean. Sci., 25, 801-812, doi: 10.3319/TAO.2014.07.07.01(A)

\section{INTRODUCTION}

The radio occultation $(\mathrm{RO})$ technique has been used to explore the atmosphere of planets in the solar system (Fjeldbo et al. 1971; Woo and Ishimaru 1974; Berman and Ramos 1980). Investigating the earth's atmosphere using the global positioning system (GPS) with the RO technique becomes promising. As shown in Fig. 1 the GPS signal is bent and delayed due to the atmospheric refractive index gradient, and received by the low earth orbit (LEO) satellite. The bending angle ( $\alpha$ in Fig. 1) is the angle between the signal vector transmitted from the GPS satellite and received by the LEO

\footnotetext{
* Corresponding author

E-mail:yusn2845@gmail.com
}

satellite. Since the signal propagation is affected by the atmosphere, many atmospheric parameters such as temperature and pressure are embedded in the signal characteristics. Therefore, many retrieval algorithms have been developed to retrieve atmospheric parameters of interest (Gorbunov and Gurvich 1998; Feng and Herman 1999; Gorbunov 2002; Igarashi et al. 2002; Jensen et al. 2003; Pavelyev et al. 2003; Kuo et al. 2004; Chiu et al. 2008). One of the important issues in developing retrieval algorithms is to understand the interaction between the GPS signal and the atmosphere. In the past wave-propagation models based on the ray tracing technique were applied in the RO signal simulation. The ray tracing technique has been applied to assess the impact of data assimilation (Zou et al. 1999; Liu and Zou 2003; Zhang 
et al. 2004) and support the RO observation (Lusignan et al. 1969; Beyerle and Hocke 2001; Yeh et al. 2012). It also has been applied to develop retrieval methods or estimate retrieval errors (Zou et al. 2002; Jensen et al. 2003; Chen et al. 2006; Li and Kiang 2011; Mannucci et al. 2011).

The ray tracing technique based on geometrical optics is used to calculate the path of the ray or the particles propagating through a system. For different applications, the assumptions of the ray tracing operators are different. When the ray tracing technique is used to support the RO observation and retrieval methods, the refractive index profiles of the earth's atmosphere is often assumed to be spherically symmetric (Beyerle and Hocke 2001; Jensen et al. 2003). Based on the spherical symmetry assumption, the Abel transform, which is an integral transform pair relating bending angle profiles and refractivity profiles (Fjeldbo et al. 1971), can be used under this assumption in the retrieval process. In such cases, the orbits of GPS and LEO satellites and the signal trajectories are assumed to be in the same plane as the earth's center.

For data assimilation, besides the retrievals of temperature and humidity (Kuo et al. 2000), the bending angle as a function of a ray impact parameter and the refractivity as a function of altitude are two additional types of GPS RO observables (Poli and Joiner 2004). The simulated bending angle and refractivity profiles can be used to assess the impact of data assimilation. For this purpose the earth's atmosphere is assumed to be nonspherically symmetric. The analytic atmosphere models, such as the National Centers for Environmental Prediction (NCEP) and the European Centre for Medium-Range Weather Forecasts (ECMWF), are used in the ray tracing operator (Zou et al. 1999, 2002; Liu and Zou 2003; von Engeln et al. 2003; Zhang et al. 2004; Chen et al. 2006) with the elliptical earth. Nevertheless, the initial and end points of the signal in the former ray tracing operators are not considered to correspond to the position of the GPS and LEO satellites.

A ray tracing model that conforms to the realistic RO signal propagation is developed. In order to ensure that the signal propagation started at the initial point and stopped close to the prescribed end point, two aiming techniques are developed in the algorithm to control the initial direction of the signal at the initial point. Furthermore, the shape of the earth is assumed as an ellipse and ECMWF analysis, which is a global coverage analysis model, is used to describe the structure of the atmosphere. The initial and end points, which are fixed to the position of GPS and LEO satellites, are recorded in Formosat-3/COSMIC (Liou et al. 2007) RO observation data. During signal trajectory integration the refractive index at the given point in the atmosphere is calculated from the near ECMWF grids using cubic spline. The simulation output is the excess signal phase which is the basic parameter of RO observations. The retrieval method in Chiu et al. (2008) is used to retrieve the bending angle profiles from the ray tracing procedure simulation results. In order to examine and demonstrate the algorithm, the ideal structure of spherically symmetric atmosphere is used to verify the ray tracing model and the ECMWF analysis with the earth's flattening is used to demonstrate the aiming algorithms.

The ray tracing technique is first developed in section 2 . In section 3, the comparisons of simulation and retrieval results are displayed, followed by the conclusions.

\section{RAY TRACING TECHNIQUE}

In an RO event the signals propagate through only a small portion of the atmosphere, with the transmitting and receiving positions recorded. The GPS signals are more strongly affected by only the local atmospheric events, such as typhoons and gravity waves. Therefore, in order to study the effect of particular atmospheric events on the received RO signals, the ray tracing model based on ECMWF analysis is developed to simulate the electromagnetic wave propagation in the earth's atmosphere. The model development will be described and explained in the following.

\subsection{Procedure of the Ray Tracing Technique}

Figure 2 shows a flowchart of the proposed ray tracing procedure. The main procedure input parameters are the times for the RO events, the initial and end points of the signal trajectories which correspond to the GPS and LEO satellite positions. By running the ray tracing procedure, the bending angle profile of the GPS signal propagating in the atmosphere can be obtained. The ray tracing procedure input parameters can be found in atmospheric atmPhs files provided by the Taiwan Analysis Center for COSMIC (TACC). In the procedure, the signal trajectories will first be deduced and each trajectory that starts at the initial point must stop close to the prescribed end point. Therefore, the simulated trajectories will correspond to the atmPrf file measurement results provided by TACC. The first step in the flowchart is to set up the refractive index distribution in the earth atmosphere based on the ECMWF analysis. Since the ECMWF analysis is updated four times per day it is able to provide atmospheric parameter profiles close to the time when the RO events took place. More details regarding ECMWF analysis application will be given in section 2.2 of this section.

The signal trajectories of the RO events are deduced and simulated in steps (2) - (6) in the flow chart. It is difficult for the simulated trajectory to end exactly at the prescribed end point due to the numerical errors and complex atmosphere. In step (2), a tolerance region centered at the prescribed end point is used in the procedure. The simulated end points are controlled to fall within the tolerance region. The geocentric distance of the simulated end point is the same as the geocentric distance of the prescribed end point. The tolerance region details will be described in section 2.3 of this section. Step (3) 
is about the signal propagation simulation in the earth's atmosphere based on the geometrical optics. The details of step (3) will be described in section 2.4 of this section.

The GPS signal trajectories will also be deduced. At the initial point, due to the wide antenna beam width the signals are emitted in many directions and generally only one of the emitted signals will be received by the LEO satellite. In the simulation, it is difficult to determine the initial propagation direction that will lead the signal to the prescribed end point (position of LEO satellite). Due to the nonspherical symmetry of the earth's atmosphere it is difficult to match the simu- lated end point exactly with the prescribed end point, especially when the signal propagates through the atmosphere at low altitude. In steps (4) - (5), two aiming algorithms are applied to determine the initial signal trajectory and control the simulated end point to fall within the tolerance region of the prescribed end point. The details of steps (4) - (5) will be described in section 2.5 of this section. When the simulated end point in steps (3), (4), or (5) falls within the prescribed end point tolerance region the procedure proceeds to step (6) to calculate the excess trajectory phase and then goes back to step (3) to deduce the next signal trajectory. The details

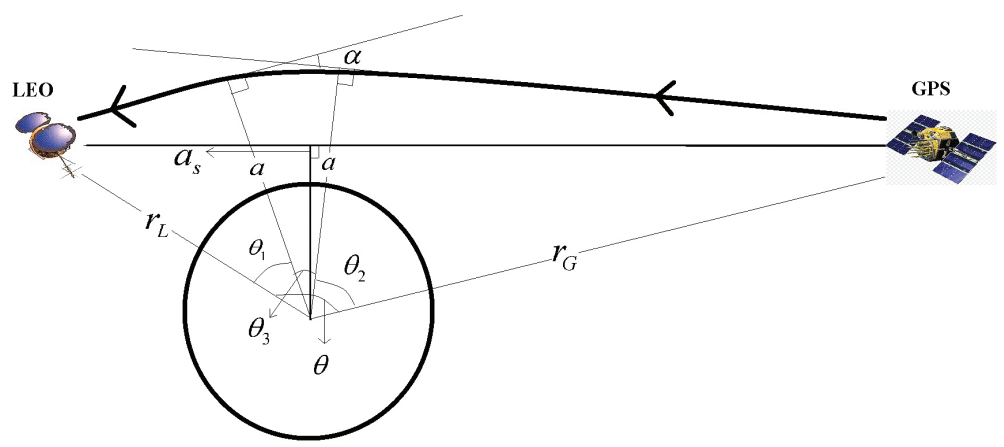

Fig. 1. Diagram of radio occultation. The thick line represents the signal from GPS satellite, propagating through atmosphere and bended by the refractivity gradient of atmosphere, and then received by LEO satellite.

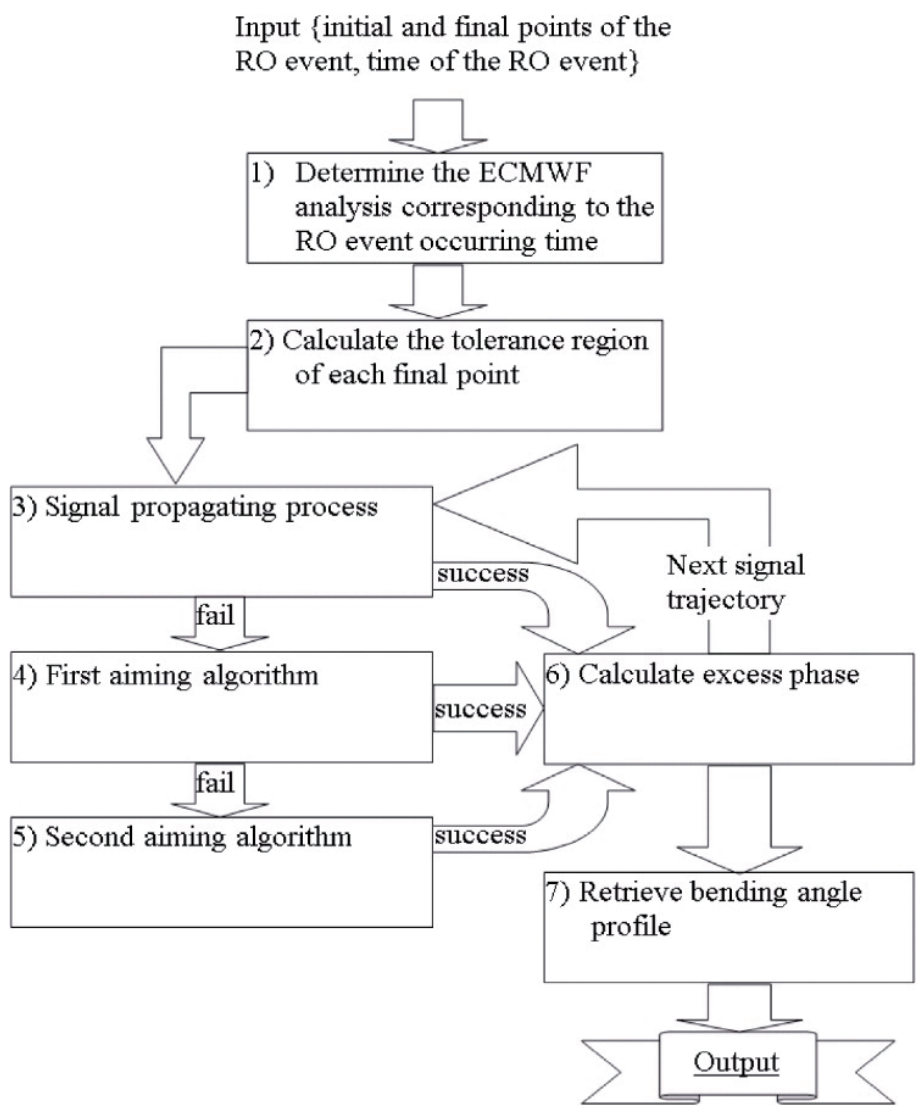

Fig. 2. Flowchart of the proposed ray tracing technique. 
of step (6) will be described in section 2.4 of this section. After simulating all trajectories for the RO event the procedure proceeds to step (7) to retrieve the bending angle profile described in section 3 .

\subsection{Setup of Atmosphere Refractive Index Based on ECMWF Analysis}

The first step in conducting this simulation is to set up the refractive index distribution in the atmosphere. Earth flattening is considered in the simulation (Syndergaard 1998). Because the earth is spinning the shape of the earth is oblate which causes the local curvature center of refractivity contours around the globe to vary with respect to the center of the earth. An ellipsoid can be used to represent the geoid shape due to gravity and hydrostatic balance (Kursinski et al. 2000). The earth's shape in this research is assumed to be an ellipsoid. The semi major and semi minor axes are set to 6378.16 and $6356.77 \mathrm{~km}$, respectively. The setup of the refractive index in the atmosphere is based on the ECMWF analysis. Since the ECMWF analysis is updated four times per day, the analysis that is nearest the RO event occurrence time is chosen for the simulation. In the ECMWF spatial coverage a coordinate grid is set up on the earth's surface from $0.0^{\circ}-358.5^{\circ} \mathrm{E}$ and $90^{\circ} \mathrm{N}-90^{\circ} \mathrm{S}$. The grid cells are 1.5 $\times 1.5$ degree. The information given at each grid point contains 37 pressure levels and the corresponding temperature and altitude. The information is updated 4 times per day. With the given pressure and temperature, the refractive index is calculated using (Thayer 1974),

$$
N=77.604 \frac{P}{T}+64.79 \frac{e}{T}+3.776 \times 10^{5} \frac{e}{T^{2}}
$$

$n=1+N \times 10^{-6}$

where $N, P, T, e$, and $n$ are the refractivity, dry pressure $(\mathrm{mb})$, temperature $(\mathrm{K})$, wet pressure $(\mathrm{mb})$, and refractive index of the atmosphere, respectively. We neglect the wet pressure and the refractive index is assumed to be 1 when the altitude is over $100 \mathrm{~km}$. When the altitude is lower than $100 \mathrm{~km}$, the refractive index is obtained by interpolating or extrapolating the discrete refractive profiles calculated from ECMWF analysis.

\subsection{Tolerance Region}

Figure 3 shows the diagrammatic tolerance region used in the simulation. The crosses in Fig. 3 are the prescribed end points, which correspond to the positions of the LEO satellite along the orbit. The LEO satellite receives a periodic GPS signal. For example, the satellites in Formosat-3/COSMIC mission record the GPS signal information every other $0.02 \mathrm{sec}$. Therefore the distance between two adjacent prescribed end points is approximately the same. The tolerance region for each prescribed end point is set to be a sphere centered at the prescribed end point. The maximum tolerable radius of each sphere is half the distance between two adjacent prescribed end points, as shown in Fig. 3. If the sphere radius is larger than the maximum tolerable radius, the angle $\theta$, which is the geocentric angle between the GPS satellite and the simulated end point, may not vary monotonically, causing calculation problems in the retrieval process. We use the maximum tolerable radius for the tolerance region.

\subsection{Signal Propagating Process}

After refractive-index distribution setup the procedure proceeds to determine the signal trajectory with the prescribed initial and end points. In this process, the trajectory with the highest altitude is first simulated because of the atmosphere effect on signal propagation at high altitude is less than that at low altitude. As mentioned in section 2.2 of this section, the atmosphere effect is considered only from $0-100 \mathrm{~km}$ in altitude. Therefore, the highest altitude of the atmosphere is set to be $100 \mathrm{~km}$.

In the algorithm, the signal propagation follows geometric optic rules. The relation between the position vector and refractive index is given by Born and Wolf (1999),

$\frac{d}{d s}\left(n \frac{d \vec{r}}{d s}\right)=n \frac{d^{2} \vec{r}}{d s^{2}}+\frac{d n}{d s} \frac{d \vec{r}}{d s}=\nabla n$

where $\vec{r}$ is the position vector of the signal trajectory, and $s$ is the trajectory length of the signal. From Eq. (3), the second-order derivative of $\vec{r}$ is

$\frac{d^{2} \vec{r}}{d s^{2}}=\frac{1}{n}\left[\nabla n-\frac{d n}{d s} \frac{d \vec{r}}{d s}\right]$

From Eq. (4), three scalar equations corresponding to the three components of $\vec{r}$ in the Cartesian coordinate are given by

$\frac{d^{2} x}{d s^{2}}=\frac{1}{n}\left[\frac{\partial n}{\partial x}-\frac{d n}{d s} \frac{d x}{d s}\right]$

$\frac{d^{2} y}{d s^{2}}=\frac{1}{n}\left[\frac{\partial n}{\partial y}-\frac{d n}{d s} \frac{d y}{d s}\right]$

$\frac{d^{2} z}{d s^{2}}=\frac{1}{n}\left[\frac{\partial n}{\partial z}-\frac{d n}{d s} \frac{d z}{d s}\right]$

where $\partial n / \partial x, \partial n / \partial y$, and $\partial n / \partial z$ are the three component of $\nabla n$. If the initial point and initial direction of the trajectory (the tangential vector of the trajectory at the initial point) are known in advance, the signal trajectory path can be obtained. 


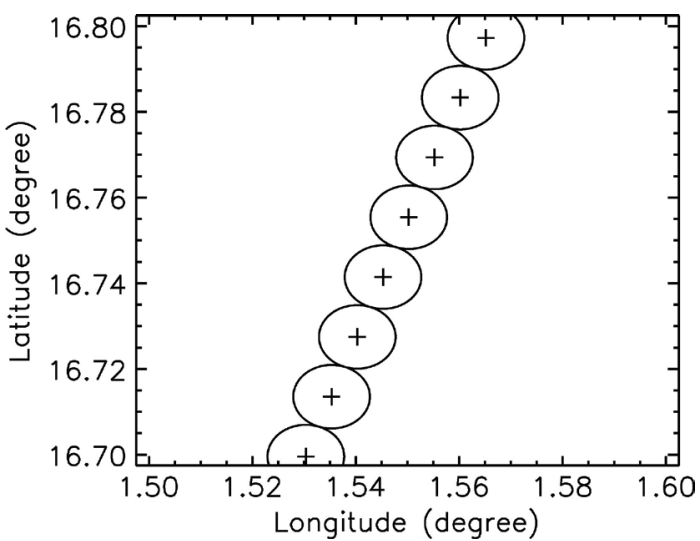

Fig. 3. The diagram of the tolerance region. The crosses denote the position of prescribed end points and the circles around the crosses are the tolerance region of associated end points. The ordinate and abscissa axis are the ellipsoidal longitude and latitude.

However, since the signals are radiated in many directions due to the wide antenna beam width, it cannot be known in advance which initial direction will lead the signal trajectory to the prescribed end point. Therefore, aiming techniques, which will be described later, are developed to search for the correct initial direction. With the aiming techniques the signal trajectory will be deduced iteratively. In the $i$-th simulation of the iteration, the trajectory is described by the set of $N+1$ points: $\left\{P_{j}^{i}\right\}$, where $i$ indicates the $i$-th iteration, and $j$ denotes the $j$-th point in the trajectory and $0 \leq j \leq N_{i}$. Therefore, $P_{0}^{i}$ and $P_{N_{i}}^{i}$ denote the initial and simulated end points, respectively, in the $i$-th iteration. The simulation of the signal trajectory is an iterative process, and it will continue until the simulated end point is located within the tolerance region of the prescribed end point. Figure 4 depicts the simulation of the $i_{t h}$ signal trajectory. The value of $\partial n / \partial x, \partial n / \partial y, \partial n / \partial z$, and $d n / d s$ at $P_{j}^{i}$ have to be known to obtain the position of $P_{j+1}^{i}$. The calculation of $\partial n / \partial x, \partial n / \partial y, \partial n / \partial z$, and $d n / d s$ are shown in Fig. 5 and the equations are as follows.

$\frac{\partial n}{\partial x}=\frac{\left[n\left(P_{j \times 1}^{i}\right)-n\left(P_{j \times 2}^{i}\right)\right]}{d s}$

$\frac{\partial n}{\partial y}=\frac{\left[n\left(P_{j y 1}^{i}\right)-n\left(P_{j y 2}^{i}\right)\right]}{d s}$

$\frac{\partial n}{\partial z}=\frac{\left[n\left(P_{j z 1}^{i}\right)-n\left(P_{j z 2}^{i}\right)\right]}{d s}$

$\frac{d n}{d s}=\frac{\left[n\left(P_{j}^{i}\right)-n\left(P_{j-1}^{i}\right)\right]}{d s}$

Substituting Eqs. (6) into (5) and solving Eq. (5) using the Runge-Kutta method (Press et al. 1992), the position of $P_{j+1}^{i}$ can be obtained.
To perform the simulation mentioned above, it is necessary to calculate the refractive index at any given point in the atmosphere. Figure 6 shows that the given position $P_{j}^{i}$ and the 16 adjacent grid points in ECMWF analysis. The refractive index calculation process is described as follows:

(1) Calculate the distance from $P_{j}^{i}$ to the earth center.

(2) Choose the 16 adjacent grid points, as shown in Fig. 6 . Calculate the altitude at each grid point by using the same distance obtained in (1). Due to the earth's flattening, the altitude of each grid point is different.

(3) If the altitude of each grid point is lower than the highest altitude provided by ECMWF analysis, the refractive index is interpolated by the 37-points refractive profiles from ECMWF analysis using the cubic spline method (Press et al. 1992). If the altitude is higher than the highest altitude provided by ECMWF analysis, the refractive index is exponentially extrapolated by the first and second highest altitudes provided by the 37 points refractive profiles calculated from ECMWF analysis.

(4) With the refractive indices at all 16 grid points, the refractive index at $P_{j}^{i}$ will be calculated by using the bicubic interpolation (Press et al. 1992).

After the signal trajectory is determined the phase path of the signal in $\mathrm{RO}$ is calculated using,

$L=\int_{G P S}^{L E O} n d s$

where $s$ is the signal trajectory length and $L$ is phase path. The excess signal phase in $\mathrm{RO}$ is calculated using

$\Delta L=L-\left|r_{L}-r_{G}\right|$

where $r_{L}$ and $r_{G}$, as shown in Fig. 1, are the LEO and GPS satellite position vectors, respectively. When the simulated end point falls within the tolerance region and does not match the LEO satellite position, $r_{L}$ in Eq. (8) is the simulated end point position vector used to calculate the excess phase.

\subsection{Aiming Algorithms}

In the first iteration of the trajectory simulation, the initial GPS signal direction is set to the direction from the initial point to the prescribed end point, vector $\overrightarrow{I A}$ as shown in Fig. 4. The signal trajectory is not a straight line due to refraction by the inhomogeneous atmosphere, so the simulated end point generally will not fall within the prescribed end point tolerance region. Two aiming algorithms will be developed and used to adjust the initial direction. The first aiming algorithm is described as follows.

(1) In $i$-th iteration shown in Fig. 4, the initial direction and the simulated end point are denoted as $\hat{t}_{0}^{i}$ and $P_{N}^{i}$, respectively. 
(2) Find the vector $\overrightarrow{\mathrm{IS}}$ as the vector from $P_{0}^{i}$ to $P_{N}^{i}$, and $\overrightarrow{\mathrm{IA}}$ from $P_{0}^{i}$ to the prescribed end point. Calculate the vector $\bar{\varphi}$ as $\bar{\varphi}=\overrightarrow{\mathrm{IS}} /|\overrightarrow{\mathrm{IS}}|-\overrightarrow{\mathrm{IA}} /|\overrightarrow{\mathrm{IA}}|$.

(3) Adjust the vector of the initial direction with $\bar{\varphi}$, as $\hat{t}_{0}^{i+1}=\left(\hat{t}_{0}^{i}-\bar{\varphi}\right) /\left|\hat{t}_{0}^{i}-\bar{\varphi}\right|$. Then start the $(i+1)$-th iteration and check if $P_{N}^{i+1}$ is in the tolerance region of the prescribed end point.

The first aiming algorithm is ceased based on the following two conditions: (1) the simulated end point is located within the tolerance region; (2) incorrect simulated end points surround the prescribed end point. The first aiming algorithm will keep running at first. If condition (1) cannot be met and condition (2) is met, the iteration is then stopped and the second aiming algorithm is activated. Condition (2) is used to ensure that the two dimensional interpolation in the second aiming algorithm can work. At low altitude the refractive index varies more rapidly than it does at high altitude. The first algorithm could fail after much iteration. The second aiming algorithm is illustrated in Figs. 7 and 8. The symbols in Fig. 7 are the directions of the initial vectors used in the second aiming algorithm process. The asterisk and the solid line in region Fig. 8 are the coordinates for the prescribed end point and the tolerance region, respectively. The other symbols are the corresponding simulated end points in Fig. 7.

The second aiming algorithm process is described as follows.

(1) Since the prescribed end point is surrounded by incorrect simulated end points using the first aiming algorithm, the correct initial vector should also be surrounded by incorrect initial vectors. In Fig. 7, the direction of the incorrect simulated end points was determined using the first aiming algorithm, denoted by the triangle symbols forming a dashed-line rectangular area from their maximum and minimum locations on the axes. The dashedline rectangular area can be used to create $\mathrm{n} \times \mathrm{n}$ grid cells to contain the directions of the initial vectors. In this study $\mathrm{n}=8$ and the directions of the initial vectors are denoted by the cross symbols in Fig. 7 .

(2) After simulating the signal trajectories using each initial vector the distance between the prescribed and simulated end points can be calculated, which are the distances between the asterisk and crosses in Fig. 8. The relation between the initial vector and the distance from the simulated and prescribed end points is obtained using bicubic interpolation (Press et al. 1992).

(3) The relation between initial vector and the distance from the simulated and prescribed end points is used to calculate the bicubic interpolation to find the minimum distance between the simulated and prescribed end points. The corresponding direction of the initial vector is used with the minimum distance to simulate the signal trajectory and find the simulated end point. The plus signs in Figs. 7 and 8 are the final initial vector direction and the corresponding simulated end point, respectively.

(4) If the simulated end point of the final initial vector is not located in the tolerance region, like the plus sign in Fig. 8 is not located in the solid line region, the process can be applied more than twice. The surrounding region of the directions of the initial vectors should be smaller. The squares in Figs. 7 and 8 are the directions of the initial vectors and the coordinates of corresponding simulated end points in second time process, respectively. The diamonds in Figs. 7 and 8 are the final initial vector direction and corresponding simulated end point of second time process, respectively. The simulated end point of second time process is located in the tolerance region.

\section{RESULTS AND COMPARISON}

The ray tracing simulation procedure described previously will be demonstrated using two cases with different atmospheric structures. In addition to the simulated signal trajectories for $\mathrm{RO}$, the corresponding parameter profiles will be retrieved and compared with other results. The two atmospheric structures are:

(1) The spherical symmetry atmosphere. In this simple case, the validity of the simulation can be preliminary examined.

(2) The atmosphere described by the ECMWF analysis was recorded on the $172^{\text {th }}$ day of 2008 . The retrieved profiles

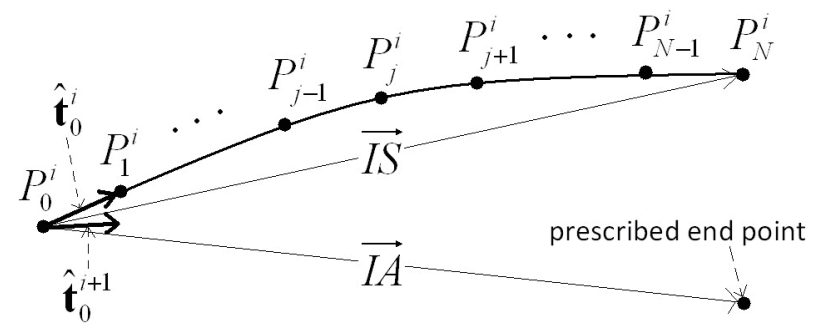

Fig. 4. Simulation of the $i_{t h}$ signal trajectory in one RO event.

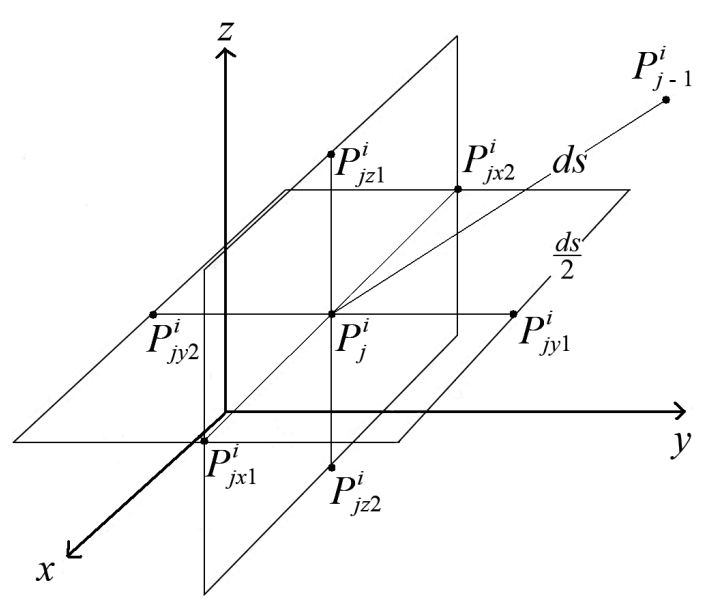

Fig. 5. Diagram for calculating the value of Eq. (6). 
(a)

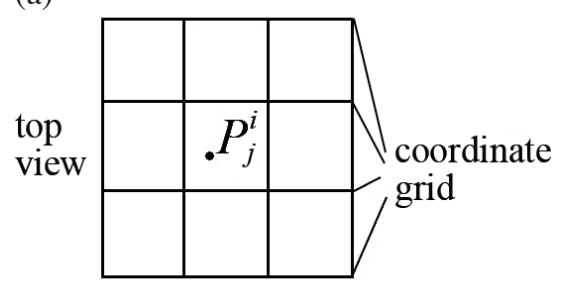

(b)

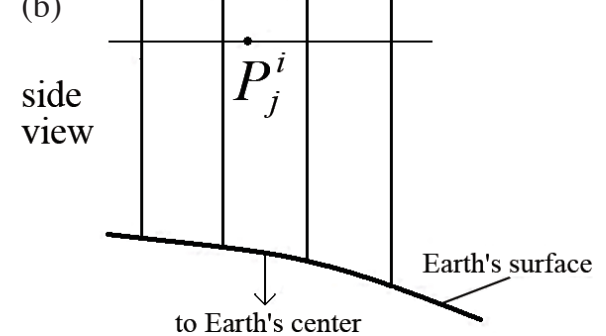

Fig. 6. Top view (a) and side view (b) of the diagram to calculate the refractive index at the simulation point.
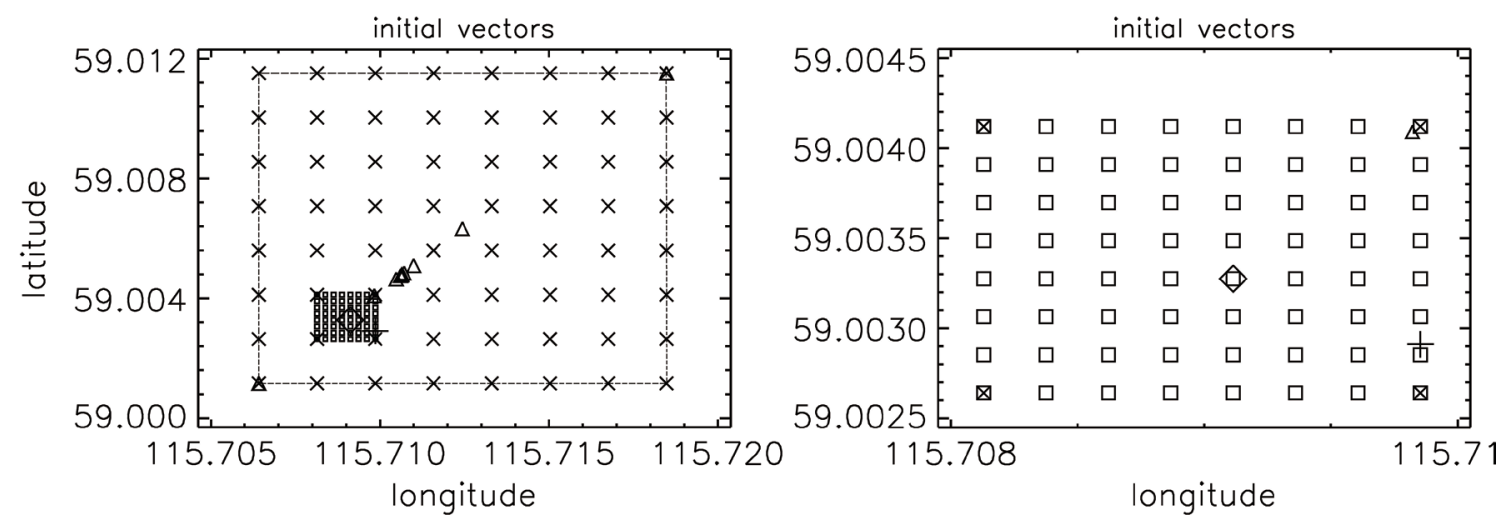

Fig. 7. The directions of the initial vectors used in the second aiming algorithm. The triangles are the directions of the initial vectors of the first aiming algorithm. The crosses and squares are the directions of the initial vectors of the first and second processes in the second aiming algorithm, respectively. The plus and diamond are the final directions of the initial vectors of the first and second processes in the second aiming algorithm, respectively.
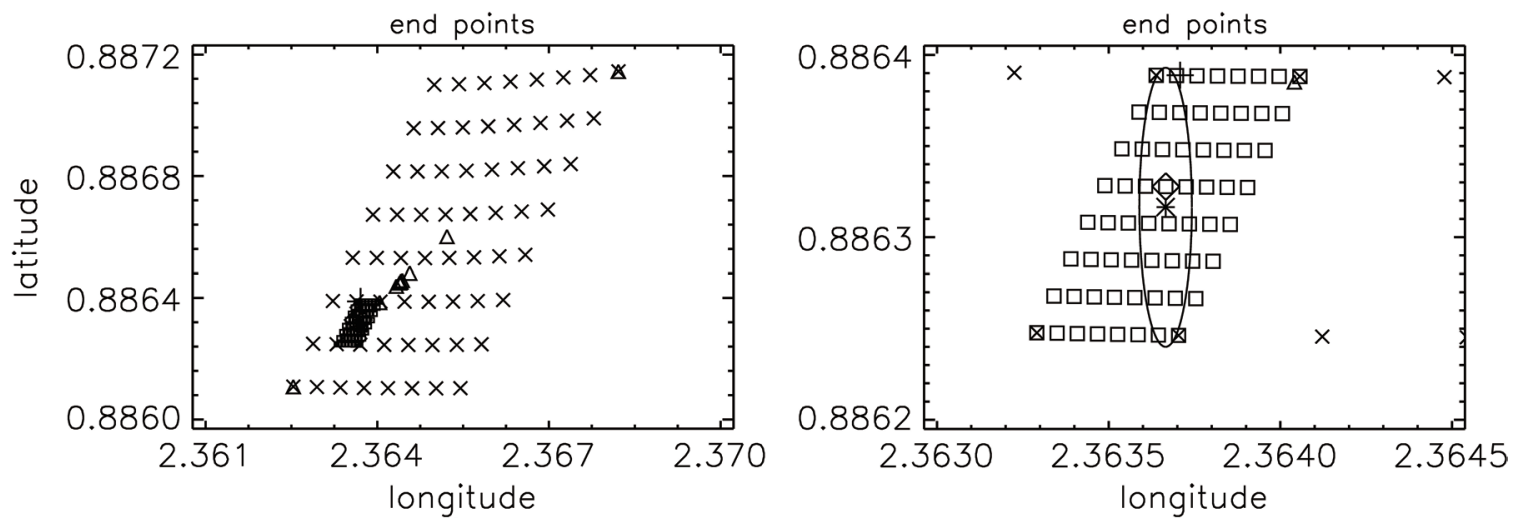

Fig. 8. The coordinate of the prescribed end point, the tolerance region, and the simulated end points in the second aiming algorithm. The asterisk and the thick-lined region are the prescribed end point and the tolerance region. The other symbols are the corresponding simulated end points of the initial vectors in Fig. 7. The directions of the initial vectors and its corresponding simulated end points have similar signs in Figs. 7 and 8.

of the bending angle will be compared with 4 profiles retrieved from the data recorded by Formosat-3/COSMIC satellites in the same day.

The first case is used to verify the ray tracing model and the second case is used to demonstrate the aiming algorithms. In these two cases, profiles of atmospheric parameter will be retrieved from the excess phase obtained from the ray tracing simulation.

\subsection{Numerical Test with Spherically Symmetric Atmosphere}

A case of spherically symmetric atmosphere is applied in the ray tracing algorithm. In the simulation, as shown in Fig. 9, the shape of the earth is a sphere and the atmospheric refractivity $(N)$ is described by the following equation (Jensen et al. 2003), 


$$
N(h)=400 \exp \left(\frac{-h}{8}\right)
$$

Where $h$ is the altitude in kilometers. The altitude range in the atmosphere is $0-100 \mathrm{~km}$. The GPS and LEO satellite altitudes are set at 20200 and $750 \mathrm{~km}$, respectively. The excess phase is simulated following the signal propagating process in section 2.2 and 2.4. The retrieval process in Chiu et al. (2008) is used to retrieve the refractivity profile using the simulated excess phase. The first step to retrieve the bending angle profile is to obtain the impact parameter by using the relation between the phase path of the signal and the impact parameter (Jensen et al. 2003)

$d L=a d \theta+\frac{\sqrt{r_{G}^{2}-a^{2}}}{r_{G}} d r_{G}+\frac{\sqrt{r_{L}^{2}-a^{2}}}{r_{L}} d r_{L}$

where $L$ is the phase path of signal, $a$ is the impact parameter, and $\theta$ is the geocentric angle between $r_{G}$ and $r_{L}$, respectively, as shown in Fig. 1. From the RO signal propagation diagram in Fig. 1, it is found that $\alpha=\theta_{3}$, where $\alpha$ is the bending angle, and $\theta_{3}=\theta-\left(\theta_{1}+\theta_{2}\right)$. Finally, the bending angle can be calculated using $\alpha=\theta-\left[\cos ^{-1}\left(\frac{a}{r_{L}}\right)+\cos ^{-1}\left(\frac{a}{r_{G}}\right)\right]$

After retrieving all bending angle of all signal trajectories, the bending angle profile is obtained. The refractive index profile can be obtained using the Abel transform (Phinney and Anderson 1968)

$n\left(a_{p}\right)=\exp \left[\frac{1}{\pi} \int_{a_{p}}^{a_{t o p}} \frac{\alpha(a)}{\sqrt{a^{2}-a_{p}^{2}}} d a\right]$

where $n\left(a_{p}\right)$ is the refractive index at $a_{p}, a_{p}$ is the impact parameter at perigee, $a_{\text {top }}$ is the maximum altitude for integration, $a$ is impact parameter, and $\alpha(a)$ is the bending angle at $a$, is applied to obtain the refractivity profile from the bending angle profile.

Figure 10 shows a comparison between the retrieved refractivity profile and the one calculated by Eq. (9). From Fig. 10, the absolute fractional difference between the real and simulated results is less than $0.1 \%$ and it indicates the ray tracing model is workable. The difference comes from the numerical errors in the integration of signal propagation and Abel transform. For example, when integrating the Abel

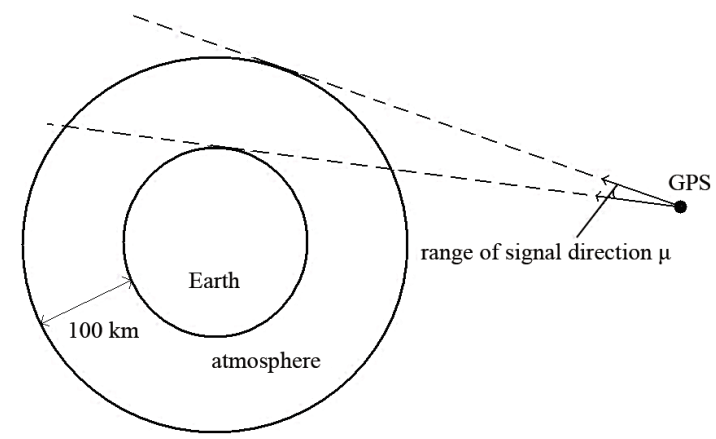

Fig. 9. The range of the altitude in atmosphere for simulation is from $0-100 \mathrm{~km}$.
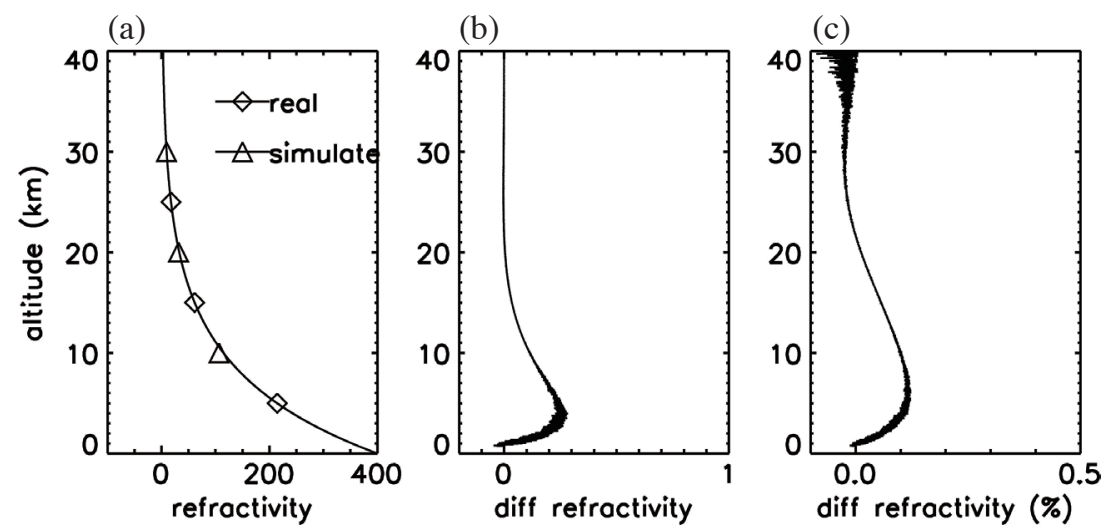

Fig. 10. Simulation results of the numerical test. Plot (a) shows the comparison of the two profiles. Plot (b) shows the difference between two profiles. Plot (c) shows the difference between two profiles in percentage. The real profile follow the refractivity of atmosphere described by Eq. (11) and the simulation profile is retrieved from the simulation result. 
transform the atmosphere is separated into $k$ layers between $a_{p}-a_{\text {top }}$. Then Eq. (12) becomes

$n\left(a_{p}\right)=\exp \left[\frac{1}{\pi} \sum_{j=1}^{k-1} \int_{a_{j}}^{a_{j+1}} \frac{\alpha(a)}{\sqrt{a^{2}-a_{p}^{2}}} d a\right]$

The relation between $a$ and $\alpha$ in the range from $a_{j}$ to $a_{j+1}$ is regarded as linear, which $\alpha(a)=\alpha\left(a_{j}\right)+q_{j}\left(a-a_{j}\right)$, where $q_{j}=\left[\alpha\left(a_{j+1}\right)-\alpha\left(a_{j}\right)\right] /\left(a_{j+1}-a_{j}\right)$. The integration term in Eq. (13) becomes

$$
\begin{aligned}
& \int_{a_{j}}^{a_{j+1}} \frac{\alpha(a)}{\sqrt{a^{2}-a_{p}^{2}}} d a \\
& \cong \int_{a_{j}}^{a_{j+1}} \frac{\alpha\left(a_{j}\right)-q_{j} a_{j}+q_{j} a}{\sqrt{a^{2}-a_{p}^{2}}} d a \\
& =\left[\alpha\left(a_{j}\right)-q_{j} a_{j}\right] \int_{a_{j}}^{a_{j+1}} \frac{1}{\sqrt{a^{2}-a_{p}^{2}}} d a+q_{j} \int_{a_{j}}^{a_{j+1}} \frac{a}{\sqrt{a^{2}-a_{p}^{2}}} d a \\
& =\left[\alpha\left(a_{j}\right)-q_{j} a_{j}\right]\left[\left.\cosh ^{-1} \frac{a}{a_{p}}\right|_{a_{j}} ^{a_{j+1}}\right]+q_{j}\left[\left.\sqrt{a^{2}-a_{p}^{2}}\right|_{a_{j}} ^{a_{j+1}}\right]
\end{aligned}
$$

The refractive index profile can then be obtained. The Abel transform error mainly comes from the linear relation assumption between $r$ and $\alpha$ in the range from $a_{j}$ to $a_{j+1}$, which is inevasible.

\subsection{Comparison with AtmPrf Data from Formosat-3/COSMIC Mission}

The 4 atmPhs file satellite information in DOY 172 of 2008 from the Formosat-3/COSMIC mission was selected first. The ECMWF analysis from the same day was used to set up the atmospheric structure in the ray tracing simulation. The atmPhs files contain the atmospheric excess phases and auxiliary data used for generating atmospheric profiles and also the observation time and GPS and LEO satellite positions. The observation time and GPS and LEO satellite positions are required to determine the position of the initial points, and prescribed end points in the ray tracing simulation. Once the signal trajectory is determined the bending angle and refractivity profiles are calculated using the retrieval procedure described in the last subsection. It is noticeable that a new local earth curvature center (Syndergaard 1998) should be defined and calculated before the retrieval procedure. The Harris method (Harris 2006) was used to determine the radius and radius vector of the local curvature. The retrieved bending angle and refractivity profiles are then compared with the raw (unoptimized) bending angle and refractivity profiles recorded in the atmPrf profiles, respectively. The atmPrf files contain the bending angle, refractivity, and dry temperature atmospheric profiles retrieved from the data in the corresponding atmPhs files.

Figure 11 shows the comparison, difference, fractional difference of the retrieved results from simulations with the corresponding retrieved results from atmPrf files, positional difference, the comparison of refractivity between the retrieved results from simulations with the corresponding retried results from atmPrf files and interpolated ECMWF profiles at the signal trajectory tangent points, and negative gradient of refractivity at the signal trajectory tangent points. The dashed lines are the retrieved bending angle and refractivity profiles. The dotted lines are the refractivity profiles derived from ECMWF at tangent points of trajectories. The positional difference is followed the equation $\left(P_{s}-P_{p}\right) / R_{t}$, where $P_{s}, P_{p}$, and $R_{t}$ are the position of the simulated end point, prescribed end point, and the radius of tolerance region. In the positional difference profiles, all of the simulated end points for the RO events are located in the tolerance region using aiming techniques. The positional difference profiles show the aiming algorithms in the model are practical. In the ray tracing technique, the default initial vector will make the simulated end points farther away from the advanced points when the signal propagates through low altitude. Furthermore, due to the complex atmospheric situation in the troposphere at low altitude, the signal propagating through the region often tend to bend toward the earth's surface causing the simulation signal to stop. Therefore the lowest altitude in simulation is only about $10 \mathrm{~km}$.

In Figs. 11a - d, the fractional difference between the simulated and atmPrf bending angle profiles higher than about $11-12 \mathrm{~km}$ in altitude is between -10 and $+10 \%$ except for the obvious peak at about $13 \mathrm{~km}$ in Figs. $11 \mathrm{c}$ and d. Below about $11-12 \mathrm{~km}$ the fractional difference becomes negative and decays to a lower fraction with altitude decrease. The decay fraction is due to the effect of water vapor. In this study, only dry pressure is considered and neglected the wet pressure. Lacking wet pressure, the refractivity is less than realistic refractivity and causes smaller bending angle. The peak at about $13 \mathrm{~km}$ in Figs. 11 c and d is caused by the violent change of refractivity. In the right panels of Figs. 11c and d, the profiles of negative gradient of refractivity have a hump at about $12-13 \mathrm{~km}$ in altitude. The violent change of refractivity causes the violent change of bending angle.

\section{CONCLUSIONS}

Ray tracing techniques have been applied to RO simulations to support retrieval algorithms and assess the impact of data assimilation. In the past, most of the applications of ray tracing techniques in RO simulation used some simplifying assumptions. For example, the earth's atmosphere is assumed to be spherically symmetric so that the Abel transform can be applied to the retrieval process. We developed a modified ray tracing model. In the model, the earth's is assumed to be an ellipse and the atmosphere is constructed using ECMWF analysis. Two aiming algorithms were developed in the model to determine the initial direction of 
(a)

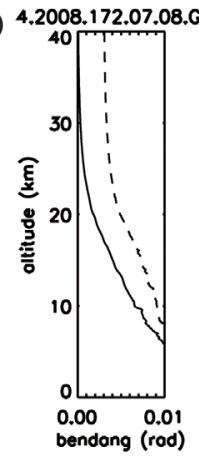

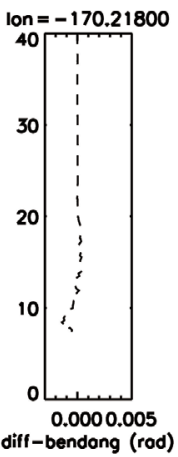
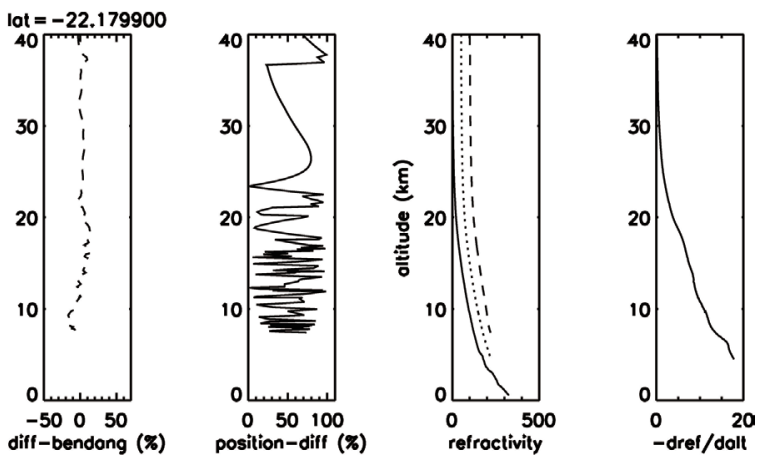

(b)
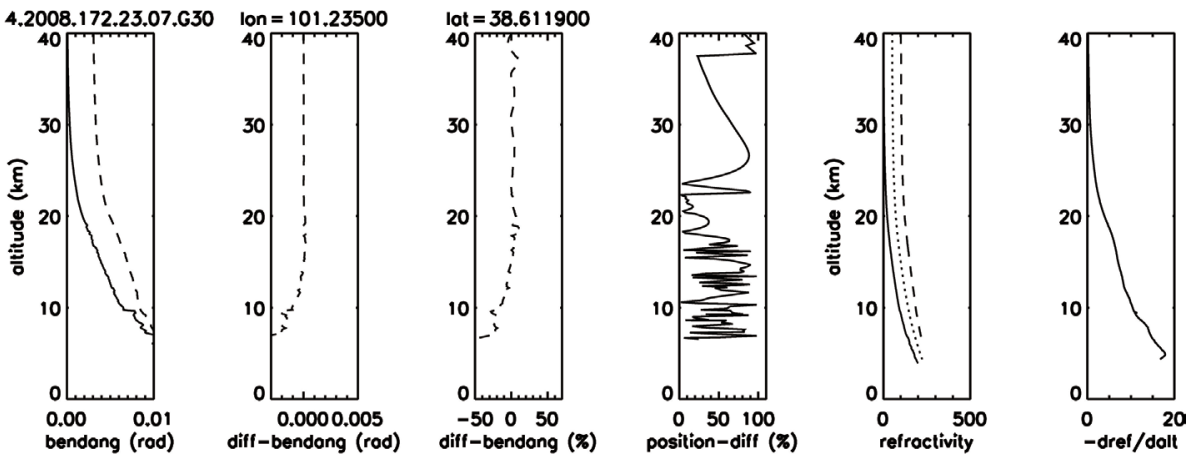

(c)
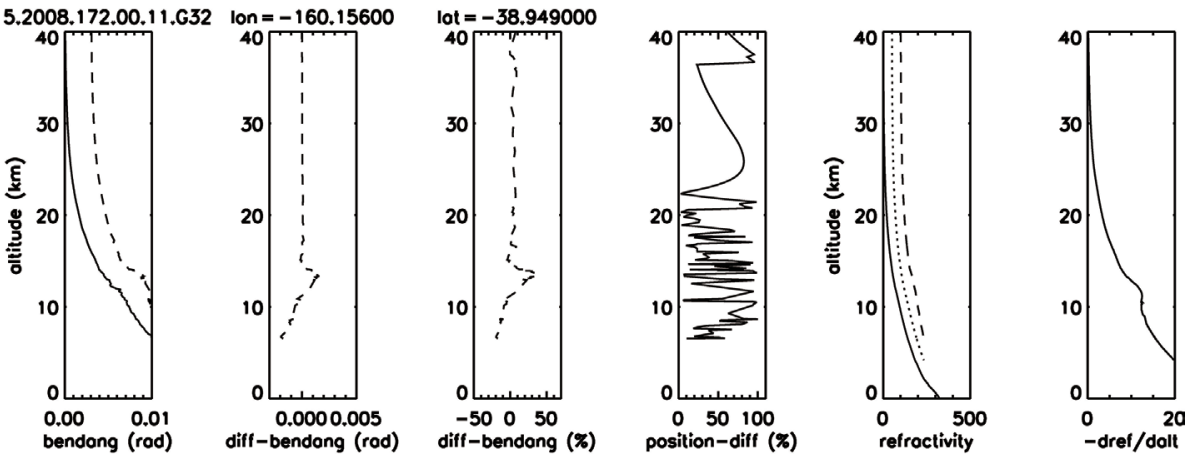

(d)
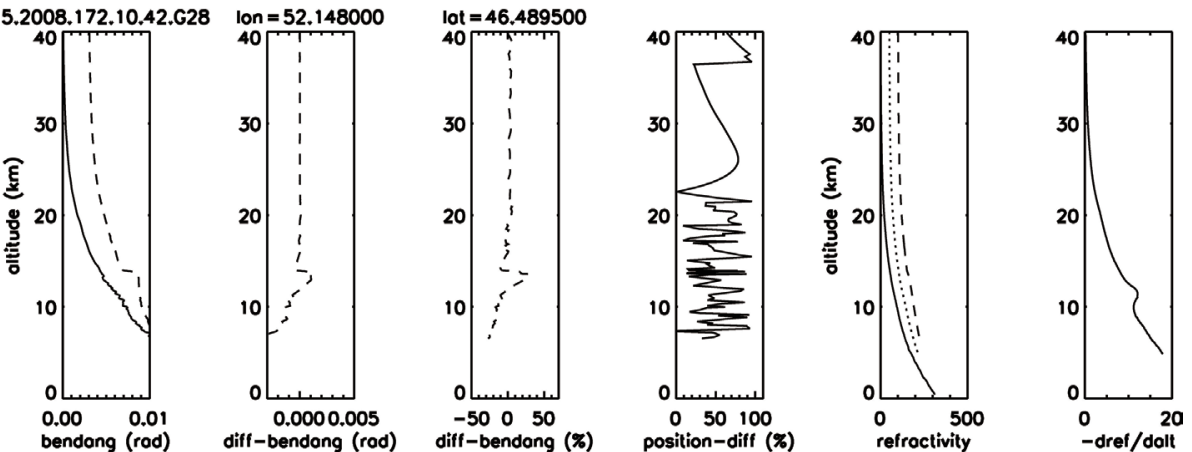

Fig. 11. The comparison results between retrieval bending angle profiles between simulation results and atmPrf files of Formosat-3/COSMIC in TACC website. The dash lines are the results of retrieval process. The solid line is the profile recorded in atmPrf file. The panels in each plot from left to right are bending angle profiles, difference of bending angle profiles, fractional difference of bending angle profile, positional difference, refeactivity, and negative gradient of refractivity at tangent points of trajectories. The dotted line is the refractivity profile derived from ECMWF. The atmPhs and atmPrf files used for simulation and comparison are atmPhs_C004.2008.172.07.08.G30_2010.2640_nc,atmPhs_C004.2008.172.23.07.G30_2010.2640_nc,atmPhs_ C005.2008.172.00.11.G32_2010.2640_nc,atmPhs_C005.2008.172.10.42.G28_2010.2640_nc,andatmPrf_C004.2008.172.07.08.G30_2010.2640_nc,atmPrf_C004.2008.172.23.07.G30_2010.2640_nc, atmPrf_C005.2008.172.00.11.G32_2010.2640_nc,atmPrf_C005.2008.172.10.42.G28_2010.2640_nc, respectively. 
the signal trajectory and make the trajectory begin at the described initial point and end within the tolerance region centered on the prescribed end point. The simulation results from the model are compared with two atmospheric cases. Based on the comparisons, the ray tracing model and the aiming algorithms are workable and can be used to simulate signal propagation in RO technique to understand the interaction between the GPS signal and the earth's atmosphere.

Acknowledgments The authors would like to thank the support from National Space Organization (NSPO), GPS Science and Application Research Center. The research is supported by grant NSC 102-2119-M-008-015, NSPO-S102037, and NSPO-S-103040.

\section{REFERENCES}

Berman, A. L. and R. Ramos, 1980: Pioneer Venus occultation radio science data generation. IEEE Trans. Geosci. Remote Sensing, GE-18, 11-14, doi: 10.1109/ TGRS.1980.350252. [Link]

Beyerle, G. and K. Hocke, 2001: Observation and simulation of direct and reflected GPS signals in radio occultation experiments. Geophys. Res. Lett., 28, 18951898, doi: 10.1029/2000GL012530. [Link]

Born, M. and E. Wolf, 1999: Principles of Optics: Electromagnetic Theory of Propagation, Interference and Diffraction of Light, $7^{\text {th }}$ edition, Cambridge University Press, New York, 985 pp.

Chen, S. H., F. Vandenberghe, and C. Y. Huang, 2006: Error characteristics of GPS retrieved refractivity using a simulation study. J. Meteorol. Soc. Jan., 84, 477-496.

Chiu, T. C., Y. A. Liou, W. H. Yeh, and C. Y. Huang, 2008: NCURO data-retrieval algorithm in FORMOSAT-3 GPS Radio-occultation mission. IEEE Trans. Geosci. Remote Sensing, 46, 3395-3405, doi: 10.1109/ TGRS.2008.2005038. [Link]

Feng, D. D. and B. M. Herman, 1999: Remotely sensing the Earth's atmosphere using the global positioning system (GPS)-The GPS/MET data analysis. J. Atmos. Ocean. Technol., 16, 989-1002, doi: 10.1175/15200426(1999)016<0989:RSTESA > 2.0.CO;2. [Link]

Fjeldbo, G., A. J. Kliore, and V. R. Eshleman, 1971: The neutral atmosphere of Venus as studied with the Mariner $\mathrm{V}$ radio occultation experiments. Astron. J., 76, 123-140, doi: 10.1086/111096. [Link]

Gorbunov, M. E. and A. S. Gurvich, 1998: Microlab-1 experiment: Multipath effects in the lower troposphere. J. Geophys. Res., 103, 13819-13826, doi: 10.1029/98JD00806. [Link]

Gorbunov, M. E., 2002: Ionospheric correction and statistical optimization of radio occultation data. Radio Sci., 37, doi: 10.1029/2000RS002370. [Link]

Harris, W. F., 2006: Curvature of ellipsoids and other surfaces. Ophthal. Physiol. Opt., 26, 497-501, doi: 10.1111/j.1475-1313.2006.00382.x. [Link]

Igarashi, K., A. Pavelyev, J. Wickert, K. Hocke, and D. Pavelyev, 2002: Application of radio holographic method for observation of altitude variations of the electron density in the mesosphere/lower thermosphere using GPS/MET radio occultation data. J. Atmos. Sol.Terr. Phys., 64, 959-969, doi: 10.1016/S1364-6826(02)00050-0. [Link]

Jensen, A. S., M. S.Lohmann, H.H. Benzon, and A. S. Nielsen, 2003: Full Spectrum Inversion of radio occultation signals. Radio Sci., 38, doi: 10.1029/2002RS002763. [Link]

Kursinski, E. R., G. A. Hajj, S. S. Leroy, and B. Herman, 2000: The GPS radio occultation technique. Terr. Atmos. Ocean. Sci., 11, 53-114.

Kuo, Y. H., S. V. Sokolovskiy, R. A. Anthes, and F. Vandenberghe, 2000: Assimilation of GPS radio occultation data for numerical weather prediction. Terr. Atmos. Ocean. Sci., 11, 157-186.

Kuo, Y. H., T. K. Wee, S. Sokolovskiy, C. Rocken, W. Schreiner, D. Hunt, and R. A. Anthes, 2004: Inversion and error estimation of GPS radio occultation data. J.Meteorol. Soc. Jpn., 82, 507-531, doi: 10.2151/ jmsj.2004.507. [Link]

Li, M. X. and J. F. Kiang, 2011: A ray tracing technique for radio occultation. 2011 IEEE International Workshop on Electromagnetics, Applications and Student Innovation (iWEM), Taipei, Taiwan, 220-222, doi: 10.1109/ iWEM.2011.6021487. [Link]

Liou, Y. A., A. G. Pavelyev, S. F. Liu, A. A. Pavelyev, N. Yen, C. Y. Huang, and C. J.Fong, 2007: FORMOSAT-3/ COSMIC GPS radio occultation mission: Preliminary results. IEEE Trans. Geosci. Remote Sensing, 45, 38133826, doi: 10.1109/TGRS.2007.903365. [Link]

Liu, H. and X. Zou, 2003: Improvements to a GPS radio occultation ray-tracing model and their impacts on assimilation of bending angle. J. Geophys. Res., 108, doi: 10.1029/2002JD003160. [Link]

Lusignan, B., G. Modrell, A. Morrison, J. Pomalaza, and S. G. Ungar, 1969: Sensing the earth's atmosphere with occultation satellites. Proc. IEEE, 57, 458-467, doi: 10.1109/PROC.1969.7000. [Link]

Mannucci, A. J., C. O. Ao, X. Pi, and B. A. Iijima, 2011: The impact of large scale ionospheric structure on radio occultation retrievals. Atmos. Meas. Tech., 4, 25252565, doi: 10.5194/amtd-4-2525-2011. [Link]

Pavelyev, A., T. Tsuda, K. Igarashi, Y. A. Liou, and K. Hocke, 2003: Wave structures in the electron density profile in the ionospheric D- and E-layers observed by radio holography analysis of the GPS/MET radio occultation data. J. Atmos. Sol.-Terr. Phys., 65, 59-70, doi: 10.1016/S1364-6826(02)00226-2. [Link]

Phinney, R. A. and D. L. Anderson, 1968: On the radio 
occultation method for studying planetary atmospheres.J. Geophys. Res., 73, 1819-1827, doi: 10.1029/ JA073i005p01819. [Link]

Poli, P. and J. Joiner, 2004: Effects of horizontal gradients on GPS radio occultation observation operators. I: Ray tracing. Q. J. R. Meteorol. Soc., 130, 2787-2805, doi: 10.1256/qj.03.228. [Link]

Press, W. H., S. A. Teukolsky, W. T. Vetterling, and B. P. Flannery, 1992: Numerical Recipes in C: The Art of Scientific Computing, $2^{\text {nd }}$ edition, Cambridge University Press, New York, 994 pp.

Syndergaard, S., 1998: Modeling the impact of the Earth's oblateness on the retrieval of temperature and pressure profiles from limb sounding. J.Atmos. Sol.-Terr. Phys., 60, 171-180, doi: 10.1016/S1364-6826(97)00056-4. [Link]

Thayer, G. D., 1974: An improved equation for the radio refractive index of air. Radio Sci., 9, 803-807, doi: 10.1029/RS009i010p00803. [Link]

Von Engeln, A., G. Nedoluha, G. Kirchengast, and S. Bühler, 2003: One-dimensional variational (1-D Var) retrieval of temperature, water vapor, and a reference pressure from radio occultation measurements: A sensitivity analysis. J. Geophys. Res., 108, doi: 10.1029/2002JD002908. [Link]
Woo, R. and A. Ishimaru, 1974: Effects of turbulence in a planetary atmosphere on radio occultation. IEEE Trans. Antennas Propag., 22, 566-573, doi: 10.1109/ TAP.1974.1140836. [Link]

Yeh, W. H., C. Y. Huang, T. Y. Hsiao, T. C. Chiu, C. H. Lin, and Y. A. Liou, 2012: Amplitude morphology of GPS radio occultation data for sporadic- $E$ layers. J. Geophys.Res., 117, A11304, doi: 10.1029/2012JA017875. [Link]

Zhang, X., Y. Liu, B. Wang, and Z. Ji, 2004: Parallel computing of a variational data assimilation model for GPS/ MET observation using the ray-tracing method. $A d v$. Atmos. Sci., 21, 220-226, doi: 10.1007/BF02915708. [Link]

Zou, X., F. Vandenberghe, B. Wang, M. E. Gorbunov, Y. H. Kuo, S. Sokolovskiy, J. C. Chang, J. G. Sela, and R. A. Anthes, 1999: A ray-tracing operator and its adjoint for the use of GPS/MET refraction angle measurements. J. Geophys. Res., 104, 22301-22318, doi: 10.1029/1999JD900450. [Link]

Zou, X., H. Liu, and R. A. Anthes, 2002: A statistical estimate of errors in the calculation of radio-occultation bending angles caused by a $2 \mathrm{D}$ approximation of ray tracing and the assumption of spherical symmetry of the atmosphere. J. Atmos. Ocean. Technol., 19, 51-64. 